\title{
Integrated Solution to Quadrotor Stabilization and Attitude Estimation Using a Pan and Tilt Camera
}

\author{
David Cabecinhas, Sérgio Brás, Carlos Silvestre, Paulo Oliveira, Rita Cunha
}

\begin{abstract}
This work proposes a cascaded architecture comprising a nonlinear attitude observer and a nonlinear controller for position and attitude stabilization of a quadrotor. The attitude estimates are obtained from rate gyros measurements, corrupted by bias, and image coordinates from a set of landmarks on the terrain, obtained by a controllable pan and tilt camera. Lateral-longitudinal stabilization is achieved with a nested saturation control law by feedback of the image measurements, estimated body attitude, and corrected rate gyros measurements. The vehicle is stabilized vertically using an additional vertical position sensor. Due to the input-tostate stability property of controller, the quadrotor position and attitude are shown to converge to the desired equilibrium point and the convergence is robust to the estimation errors. Additionally, the pan and tilt camera is actively actuated to keep the landmarks visible in the image sensor for most operating conditions. The performance of the proposed ensemble is illustrated with some simulation results.
\end{abstract}

\section{INTRODUCTION}

Over the last decades, the development of Unmanned Aerial Vehicles (UAVs) has witnessed a remarkable evolution. The operation of such autonomous vehicles indoors and in the vicinity of tall structures, where GPS signals are unreliable or simply unavailable, calls for alternative solutions based on local sensor measurements such as visionbased control [1], [2]. In this work, we propose an integrated feedback control architecture for the stabilization of a quadrotor based on visual information and attitude estimates provided by a nonlinear observer.

The literature on vision-based rigid-body stabilization and estimation highlights important questions and indicates possible solutions to i) keeping feature visibility along the system's trajectories for a large region of attraction [3], [4], ii) minimizing the required knowledge about the 3-D model of the observed object [5], iii) guaranteeing convergence in the presence of camera parametric uncertainty and image measurement noise [5], iv) establishing observability conditions for attitude estimation [6]. A variety of algebraic and iterative estimation methods based on point and line correspondences have been proposed (see for example [7]). Algorithms for attitude estimation greatly benefit from the integration with inertial sensors, namely rate gyros and

This work was partially funded by Fundação para a Ciência e a Tecnologia (FCT) projects PEst-OE/EEI/LA0009/2011, PTDC/MAR/64546/2006 OBSERVFLY and PTDC/EEA-CRO/102857/2008 SCARVE, and by the EU project TRIDENT of the EC-FP7. The work of D. Cabecinhas and $\mathrm{S}$. Brás was supported by the PhD Student grants from the FCT SFRH/BD/31439/2006 and SFRH/BD/47456/2008, respectively.

The authors are with the Department of Electrical Engineering and Computer Science, and LARSys, Universidade Técnica de Lisboa, 1049-001 Lisboa, Portugal. C. Silvestre is also with Department of Electrical and Computer Engineering, Faculty of Science and Technology of the University of Macau. \{dcabecinhas, sbras, csj,pjcro, rita\}eisr.ist.utl.pt accelerometers as well as from the use of dynamic filtering and observer techniques [8], [9], [10].

The quadrotor is a typical example of an underactuated vehicle ideally suited for the development and test of new control strategies due to its simplicity and maneuverability. The simplified model commonly adopted for both quadrotors and helicopters is a 6-DoF rigid body actuated in force and torque with four inputs given by a scalar thrust and three torque inputs. These rotorcraft have drawn the attention of the control community in recent years. Several approaches have concentrated on using nonlinear techniques, such as backstepping [11], [12] and feedforward control [13], [14], to solve the trajectory tracking problem for a single vehicle.

The main contribution of this paper is an integrated solution to motion stabilization and attitude estimation based on rate gyro measurements and visual information from a set of landmarks placed on the terrain, which are retrieved by a pan and tilt camera. The proposed nonlinear observer estimates the quadrotor attitude and the rate gyros bias, driving the estimation error exponentially fast to the origin. The pan and tilt camera controller differs from other solutions present in the literature [2] as it does not require explicit estimation of the camera's position and velocity. In the proposed controller, only measurements from the available sensors and estimates from the attitude observer are used, instead of classic fullstate feedback. Notwithstanding, overall stability is still attained due to the controllers robustness and Input-to-State Stability (ISS) properties.

\section{NOMENCLATURE}

The rotation group is denoted by $\mathrm{SO}(3)=$ $\left\{R \in \mathbb{R}^{3 \times 3}: R^{T} R=I_{3}, \operatorname{det}(R)=1\right\}$, where $I_{3}$ denotes the $3 \times 3$ identity matrix, and the associated Lie algebra is denoted by $\mathfrak{s o}(3)$ and is composed by the $3 \times 3$ skewsymmetric matrices $\mathfrak{s o}(3)=\left\{K \in \mathbb{R}^{3 \times 3}: K^{T}=-K\right\}$. The skew-symmetric operator is denoted as $S(\mathbf{x}): \mathbb{R}^{3} \rightarrow \mathfrak{s o}(3)$ such that $S(\mathbf{x}) \mathbf{y}=\mathbf{x} \times \mathbf{y}=$, where $\mathbf{x}, \mathbf{y} \in \mathbb{R}^{3}$, whereas the inverse map $S^{-1}():. \mathfrak{s o}(3) \rightarrow \mathbb{R}^{3}$ is defined such that $S^{-1}(S(\mathbf{x}))=\mathbf{x}$. The notation $\operatorname{diag}(\mathbf{a})$ describes a diagonal matrix formed by placing the elements of $\mathbf{a} \in \mathbb{R}^{n}$ in the main diagonal. The Frobenius norm of matrices is denoted by $\|M\|_{F}$. Finally, $\sigma: \mathbb{R} \rightarrow \mathbb{R}$ is a saturation function that satisfies $\left|\sigma^{\prime}(x)\right|=\left|\frac{d \sigma(x)}{d x}\right| \leq 2$ for all $x, x \sigma(x)>$ 0 for all $x \neq 0, \sigma(0)=0, \sigma(x)=\operatorname{sign}(x)$ for $|x|>1$, and $|x|<|\sigma(x)|<1$ for $|x|<1$.

\section{Problem Formulation}

Consider a fixed inertial frame $\{I\}$ and a body frame $\{B\}$ attached to the vehicle's center of mass. The configuration of $\{B\}$ with respect to $\{I\}$ is given by the pair 


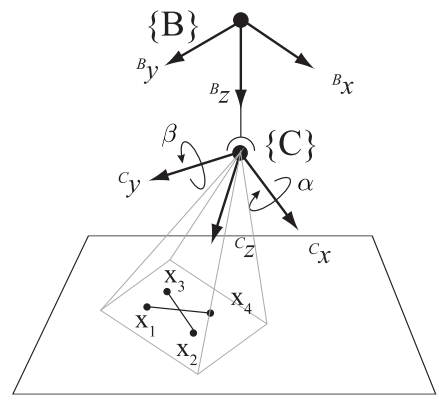

Fig. 1. Diagram of the camera and landmarks setup.

$(R, \mathbf{p})=\left({ }_{B}^{I} R,{ }^{I} \mathbf{p}_{B}\right)$. Attached to the rigid body there is a pan and tilt camera. The camera frame is denoted as $\{C\}$. Its origin coincides with the camera's center of projection, and the $z$-axis is aligned with the optical axis. The observed scene consists of four points whose coordinates in $\{I\}$ are denoted by ${ }^{I} \mathbf{x}_{i} \in \mathbb{R}^{3}, i \in 1, \ldots, 4$. Without loss of generality, the origin of $\{I\}$ is assumed to coincide with the centroid of the feature points so that $\sum_{i=1}^{4}{ }^{I} \mathbf{x}_{i}=0$ and the landmarks are assumed to belong to the $x-y$ plane. The problem setup is illustrated in Fig. 1.

\section{A. Sensor Suite}

We assume that a triad of rate gyros is installed onboard the platform and that it is aligned with $\{B\}$, so that it provides measurements of the body angular velocity $\boldsymbol{\omega}_{B}$ corrupted by a constant unknown bias term $\mathbf{b}$, such that

$$
\boldsymbol{\omega}_{r}=\boldsymbol{\omega}_{B}+\mathbf{b}, \quad \dot{\mathbf{b}}=\mathbf{0} .
$$

As shown in Fig. 1, the camera can describe pan and tilt motions corresponding to the angles $\alpha$ and $\beta$, respectively. As such, the rotation matrix from $\{C\}$ to $\{B\}$ is given by

$$
\begin{gathered}
{ }_{C}^{B} R=R_{\mathrm{pan}} R_{\mathrm{tilt}}, \\
R_{\mathrm{pan}}=R_{x}(\alpha), \quad R_{\mathrm{tilt}}=R_{y}(\beta)
\end{gathered}
$$

where $R_{x}(\cdot)$ and $R_{y}(\cdot)$ denote rotation matrices about the $x$-axis and $y$-axis, respectively. We denote the configuration of $\{C\}$ with respect to $\{I\}$ by $\left({ }_{C}^{I} R,{ }^{I} \mathbf{p}_{C}\right)$, where ${ }_{C}^{I} R$ is the rotation matrix from $\{C\}$ to $\{I\}$ and ${ }^{I} \mathbf{p}_{C}$ the position of the origin of $\{C\}$ with respect to $\{I\}$. Then, the 3 -D coordinates of the feature points expressed in $\{C\}$ can be written as $\mathbf{q}_{i}={ }_{C}^{I} R^{T I} \mathbf{x}_{i}+{ }^{C} \mathbf{p}_{I}, i \in 1, \ldots, 4$, where ${ }^{C} \mathbf{p}_{I}=-{ }_{I}^{C} R^{I} \mathbf{p}_{C}$, and, using the perspective camera model [8], the 2-D image coordinates of those points $\mathbf{y}_{i} \in \mathbb{R}^{2}$ can be written as

$$
\left[\begin{array}{ll}
\mathbf{y}_{i}{ }^{T} & 1
\end{array}\right]^{T}=\delta_{i} A \mathbf{q}_{i}
$$

where $A \in \mathbb{R}^{3 \times 3}$ is the camera calibration matrix, assumed to be known, and $\delta_{i}$ is an unknown scalar encoding depth information and given by $\delta_{i}=\left(\mathbf{u}_{3}^{T} \mathbf{q}_{i}\right)^{-1}, \mathbf{u}_{3}=\left[\begin{array}{lll}0 & 0 & 1\end{array}\right]^{T}$.

\section{B. Quadrotor Model}

We model the quadrotor vehicle as a rigid body that is actuated in force and torque. The kinematic and dynamic equations of motion for the rigid body can be written as

$$
\begin{aligned}
\dot{R} & =R S\left(\boldsymbol{\omega}_{B}\right) \\
\dot{\mathbf{p}} & =R \mathbf{v} \\
\dot{\boldsymbol{\omega}}_{B} & =-\mathbb{J}^{-1} S\left(\boldsymbol{\omega}_{B}\right) \mathbb{J} \boldsymbol{\omega}_{B}+\mathbb{J}^{-1} \mathbf{n} \\
\dot{\mathbf{v}} & =-S\left(\boldsymbol{\omega}_{B}\right) \mathbf{v}+\frac{1}{m} \mathbf{f},
\end{aligned}
$$

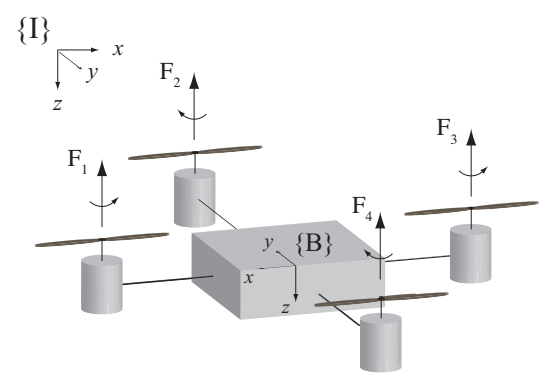

Fig. 2. Quadrotor vehicle setup.

where the position $\mathbf{p}$ is expressed in the inertial frame $\{I\}, R$ is the rotation matrix from $\{B\}$ to $\{I\}$, and the angular velocity $\boldsymbol{\omega}_{B} \in \mathbb{R}^{3}$ and the linear velocity $\mathbf{v} \in \mathbb{R}^{3}$ are expressed in the body frame $\{B\}$. The scalar $m$ and the matrix $\mathbb{J} \in \mathbb{R}^{3 \times 3}$ represent the quadrotor's mass and moment of inertia, $\mathbf{f} \in \mathbb{R}^{3}$ and $\mathbf{n} \in \mathbb{R}^{3}$ denote respectively the external force and torque expressed in the body frame. Aerodynamic drag forces due to the fuselage are neglected given the low speeds at which the quadrotor operates.

Figure 2 shows a sketch of the quadrotor setup, together with the force generated by each motor $F_{i}$ and the direction of rotation for each propeller. The bijective correspondence between the motor forces and the total thrust $T$ and torque $\mathbf{n}=\left[n_{1} n_{2} n_{3}\right]^{T}$ can be found in [12]. The external force in body coordinates is given by

$$
\mathbf{f}=-T \mathbf{u}_{3}+m g R^{T} \mathbf{u}_{3}
$$

where $\mathbf{u}_{3}=\left[\begin{array}{lll}0 & 0 & 1\end{array}\right]^{T}$ and $g$ is the gravitational acceleration.

\section{AtTItUde ObSERVER}

In this section, we present a nonlinear observer based on [15]. The proposed observer is designed to match the rigid body attitude kinematics by taking the form

$$
\dot{\hat{R}}=\hat{R} S\left(\hat{\boldsymbol{\omega}}_{B}\right),
$$

where $\hat{\boldsymbol{\omega}}_{B}$ is the feedback term designed to compensate for the estimation errors. The attitude and bias estimation errors are defined as $\tilde{R}=\hat{R} R^{T}$ and $\tilde{\mathbf{b}}=\hat{\mathbf{b}}-\mathbf{b}$, respectively. Using (3) and (7), the rotation error dynamics are given by

$$
\dot{\tilde{R}}=\tilde{R} S\left(R\left(\hat{\boldsymbol{\omega}}_{B}-\boldsymbol{\omega}_{B}\right)\right) .
$$

Some rotational degrees of freedom are unobservable when features are all collinear. The following necessary condition for attitude estimation based on image measurements is assumed.

Assumption 1: There are at least four features of which no three are collinear.

The feedback law is a function of the angular rate measurements and the image coordinates of the landmarks. To derive it, we start by defining the following matrices

$$
X=\left[\begin{array}{lll}
{ }^{I} \mathbf{x}_{1} & \ldots & { }^{I} \mathbf{x}_{4}
\end{array}\right], \quad Y=\left[\begin{array}{ccc}
\mathbf{y}_{1} & \cdots & \mathbf{y}_{4} \\
1 & \cdots & 1
\end{array}\right],
$$

where ${ }^{I} \mathbf{x}_{i}$ are the 3-D coordinates of the feature points expressed in $\{I\}$ and $\mathbf{y}_{i}$ the corresponding 2-D image coordinates. We can now state the following lemma.

Lemma 2: Let $\boldsymbol{\sigma}=\left[\begin{array}{llll}\sigma_{1} & \sigma_{2} & \sigma_{3} & \sigma_{4}\end{array}\right]^{T} \in \mathbb{R}^{4} \backslash\{\mathbf{0}\}$ and $\boldsymbol{\rho}=$ $\left[\begin{array}{llll}\rho_{1} & \rho_{2} & \rho_{3} & \rho_{4}\end{array}\right]^{T} \in \mathbb{R}^{4} \backslash\{\boldsymbol{0}\}$ be such that $Y \boldsymbol{\sigma}=\mathbf{0}, X \boldsymbol{\rho}=$ 
$\mathbf{0}$, and $\mathbf{1}^{T} \boldsymbol{\rho}=0$, where $\mathbf{1}=\left[\begin{array}{llll}1 & 1 & 1 & 1\end{array}\right]^{T}$. Consider that the features verify Assumption 1 and the camera configuration is such that the image is not degenerate (neither a point nor a line). Then, the depth variables $\delta_{i}$ can be written as

$$
\delta_{i}=\alpha \frac{\rho_{i}}{\sigma_{i}}
$$

where $\alpha \in \mathbb{R}, \rho_{i} \neq 0$, and $\sigma_{i} \neq 0$ for $i \in 1, \ldots, 4$.

Due to space constraints the proof of this lemma is omitted. However, it can be found in [15].

Writing (2) in matrix form and using Lemma 2, we have $Y=A\left({ }_{C}^{I} R^{T} X-{ }^{C} \mathbf{p}_{I} \mathbf{1}^{T}\right) \alpha D_{\sigma}^{-1} D_{\rho}$, where $D_{\rho}=\operatorname{diag}(\boldsymbol{\rho})$. From the feature centroid constraint $X \mathbf{1}=0$, it follows that

$$
\alpha_{C}^{I} R^{T} X=A^{-1} Y D_{\rho}^{-1} D_{\sigma}\left(I_{4}-\frac{1}{4} \mathbf{1 1}^{T}\right),
$$

which encodes information about the attitude of the camera up to a scale factor. We can use the properties of the rotation matrix and the positive depth constraint $\delta_{i}>0$ to obtain the normalized vector readings ${ }^{C} \overline{\mathbf{x}}_{i}={ }_{C}^{I} R^{T I} \overline{\mathbf{x}}_{i}=\operatorname{sign}(\alpha) \alpha_{C}^{I} R^{T I} \mathbf{x}_{i} /\left(\left\|\alpha_{C}^{I} R^{T I} \mathbf{x}_{i}\right\|\right)$, where $\operatorname{sign}(\alpha)=\operatorname{sign}\left(\frac{\rho_{i}}{\sigma_{i}}\right)$ and ${ }^{I} \overline{\mathbf{x}}_{i}={ }^{I} \mathbf{x}_{i} /\left\|{ }^{I} \mathbf{x}_{i}\right\|, i=1, \ldots, 4$. Note that no discontinuity is introduced by the use of the $\operatorname{sign}($.$) function.$

\section{A. Observer Design}

Consider the Lyapunov function

$$
V=\frac{\left\|\tilde{R}-I_{3}\right\|_{F}^{2}}{2}+\frac{1}{2 k_{b}}\|\tilde{\mathbf{b}}\|^{2}=\operatorname{tr}\left(I_{3}-\tilde{R}\right)+\frac{1}{2 k_{b}}\|\tilde{\mathbf{b}}\|^{2},
$$

where $k_{b}>0$. By using (8) and noting that $\operatorname{tr}(A S(\mathbf{a}))=$ $-S^{-1}\left(A-A^{T}\right)^{T} \mathbf{a}, A \in \mathbb{R}^{3 \times 3}, \mathbf{a} \in \mathbb{R}^{3}$, we obtain

$$
\dot{V}=\mathbf{s}_{\omega}^{T}\left(\hat{\boldsymbol{\omega}}_{B}-\boldsymbol{\omega}_{B}\right)+\frac{1}{k_{b}} \dot{\tilde{\mathbf{b}}}^{T} \tilde{\mathbf{b}},
$$

where $\mathbf{s}_{\omega}=R^{T} S^{-1}\left(\tilde{R}-\tilde{R}^{T}\right)$.

Consider now the following attitude feedback law

$$
\hat{\boldsymbol{\omega}}_{B}=\boldsymbol{\omega}_{r}-\hat{\mathbf{b}}-k_{\omega} \mathbf{s}_{\omega}=\boldsymbol{\omega}_{B}-\tilde{\mathbf{b}}-k_{\omega} \mathbf{s}_{\omega},
$$

where $k_{\omega}>0$. Applying (10) in (9) and defining

$$
\dot{\hat{\mathbf{b}}}:=k_{b} \mathbf{s}_{\omega}
$$

the Lyapunov function derivative becomes $\dot{V}=-k_{\omega}\left\|\mathbf{s}_{\omega}\right\|^{2}$. Considering the feedback law (10) and the update law (11), the closed loop attitude error dynamics can be written as

$$
\begin{gathered}
\dot{\tilde{R}}=-k_{\omega} \tilde{R}\left(\tilde{R}-\tilde{R}^{T}\right)-\tilde{R} S(R \tilde{\mathbf{b}}) \\
\dot{\tilde{\mathbf{b}}}=k_{b} R^{T} S^{-1}\left(\tilde{R}-\tilde{R}^{T}\right)
\end{gathered}
$$

Exploiting the results derived for Linear Time-Varying (LTV) systems in [16], it can be shown that the trajectories of the system (12) converge to the desired equilibrium point. Global asymptotic stability of the origin is however precluded by topological limitations associated with the points that verify $\left\|\tilde{R}-I_{3}\right\|_{F}^{2}=8[17]$.

Theorem 3: Assume that $\omega_{B}$ is bounded and $\dot{\mathbf{b}}=0$. Then, for any initial condition that verifies

$$
\frac{\left\|\tilde{\mathbf{b}}\left(t_{0}\right)\right\|^{2}}{8-\left\|\tilde{R}\left(t_{0}\right)-I_{3}\right\|_{F}^{2}}<k_{b}
$$

the estimation error $\tilde{\mathbf{x}}=(\tilde{R}, \tilde{\mathbf{b}})$ is bounded and $\| \tilde{R}(t)-$ $I_{3} \|_{F}^{2}<8$ for all $t \geq t_{0}$. Moreover, the attitude and bias estimation errors converge exponentially fast to the equilibrium point $(\tilde{R}, \tilde{\mathbf{b}})=\left(I_{3}, 0\right)$ for any initial condition satisfying (13).

The proof of this theorem is omitted due to space constraints. However, it can be obtained following a similar reasoning of the proof of Theorem 1 in [15].

Consider the identity $Q S^{-1}\left(A-A^{T}\right)=$ $S^{-1}\left(Q A Q^{T}-Q A^{T} Q^{T}\right)$, where $A \in \mathbb{R}^{3 \times 3}, Q \in \operatorname{SO}(3)$, and the relation ${ }_{C}^{I} R=R_{C}^{B} R={ }^{I} \bar{X}^{C} \bar{X}^{\dagger}$, where ${ }_{C}^{B} R$ is given by (1), ${ }^{C} \bar{X}=\left[{ }^{C} \overline{\mathbf{x}}_{1}, \ldots,{ }^{C} \overline{\mathbf{x}}_{4},{ }^{C} \overline{\mathbf{x}}_{i} \times{ }^{C} \overline{\mathbf{x}}_{j}\right]$, ${ }^{I} \bar{X}=\left[{ }^{I} \overline{\mathbf{x}}_{1}, \ldots,{ }^{I} \overline{\mathbf{x}}_{4},{ }^{I} \overline{\mathbf{x}}_{i} \times{ }^{I} \overline{\mathbf{x}}_{j}\right]$, for any linearly independent ${ }^{I} \overline{\mathbf{x}}_{i}$ and ${ }^{I} \overline{\mathbf{x}}_{j}$. Using the following derivation, the feedback term $\mathbf{s}_{\omega}$ can be expressed as an explicit function of the sensor readings and known quantities

$$
\mathbf{s}_{\omega}=S^{-1}\left({ }_{C}^{B} R\left({ }^{C} \bar{X}^{\dagger}\right)^{T I} \bar{X}^{T} \hat{R}-\hat{R}^{T I} \bar{X}^{C} \bar{X}_{C}^{\dagger B} R^{T}\right),
$$

where ${ }^{C} \bar{X}^{\dagger}={ }^{C} \bar{X}^{T}\left({ }^{C} \bar{X}^{C} \bar{X}^{T}\right)^{-1}$.

Remark 4: Note that the conditions of Theorem 3 are not restrictive, since $\omega_{B}$ is intrinsically bounded due to the practical limitation on the energy of the system and the condition (13) can always be verified inside the almost global domain of attraction by tuning the gains.

\section{PAN AND Tilt Controller}

In this section, we address the problem of keeping the features inside the image plane by exploring the camera's ability to describe pan and tilt angular motions. The strategy adopted to achieve this goal amounts to controlling the camera pan and tilt angular velocities, $\dot{\alpha}$ and $\dot{\beta}$, using directly the image measurements $\mathbf{y}_{i}$ and the angular velocity readings $\boldsymbol{\omega}_{r}$, so as to keep the image of the features' centroid, denoted as $\overline{\mathbf{y}}$ at a close distance from the center of the image plane.

We use the controller proposed in [15], which with the proper redefinition of axes is given by the following expression

$$
\left[\begin{array}{c}
\dot{\alpha} \\
\dot{\beta}
\end{array}\right]=k_{c}\left[\begin{array}{cc}
0 & -\frac{1}{\cos \beta} \\
1 & 0
\end{array}\right] \overline{\mathbf{y}}-\left[\begin{array}{ccc}
1 & 0 & -\tan \beta \\
0 & 1 & 0
\end{array}\right] R_{\mathrm{pan}}^{T} \hat{\boldsymbol{\omega}}_{B},
$$

where $\hat{\boldsymbol{\omega}}_{B}=\boldsymbol{\omega}_{r}-\hat{\mathbf{b}}, k_{c}>0$, and $\overline{\mathbf{y}}$ is bounded by the size of the image frame.

This controller guarantees that $\overline{\mathbf{y}}$ converges to the center of the image provided that the linear velocity of the camera and the error of the rate gyros bias converge to zero. In fact, in [15] it is shown that the controller is input-to-state stable (ISS) with respect to $\mathbf{v}$ and $q_{z} \tilde{\mathbf{b}}$, where $\mathbf{v}$ is the camera's linear velocity and $q_{z}$ the projection of the features' centroid in the $z$-axis of the camera frame. The distance between the image of the centroid and the origin is ultimately bounded by $\left\|\Pi \mathbf{v} / q_{z}\right\|$ and $\|\tilde{\mathbf{b}}\|$, where $\Pi \in \mathbb{R}^{3 \times 3}$ is the $x-y$ plane projection matrix, and it converges exponentially fast to that bound.

\section{QUADROTOR CONTROLLER}

The control objective consists of designing a control law for the quadrotor actuations $\mathbf{f}$ and $\mathbf{n}$, which ensures the convergence of the horizontal position in frame $\{I\}$ to zero with the largest possible basin of attraction, while maintaining the landmarks visible in the image sensor and the vehicle's vertical coordinate stable.

The proposed controller makes use of the unit quaternions to represent the attitude, in contrast with the rotation matrix 
parametrization used previously. Unit quaternions $\mathbf{q} \in \mathbb{S}^{4}$, are written in the form $\mathbf{q}=\left[\begin{array}{ll}q_{0} & q^{T}\end{array}\right]^{T}$, where the scalar part $q_{0} \in \mathbb{R}$ is related to the rotation angle $\theta \in[0, \pi)$ and the vector part $q=\left[\begin{array}{lll}q_{1} & q_{2} & q_{3}\end{array}\right]^{T} \in \mathbb{R}^{3}$ to the axis of rotation $\mathbf{n} \in \mathbb{S}^{3}$ through

$$
\mathbf{q}(\theta, \mathbf{n})=\left[\begin{array}{c}
q_{0} \\
q
\end{array}\right]=\left[\begin{array}{c}
\cos (\theta / 2) \\
\mathbf{n} \sin (\theta / 2)
\end{array}\right] .
$$

The methodology adopted to address the quadrotor vehicle control problem is in line with the state feedback controller proposed in [13]. However, as the full system state is not directly available for feedback, the controller is modified to use the image measurements and attitude estimates to stabilize the quadrotor position at the desired location.

\section{A. Stabilization of the Vertical Error Dynamics}

The control objective is to drive the vehicle to a given reference altitude $h^{\star}$. Let $h_{0}$ be the altitude of frame $\{I\}$. Then, the altitude of the vehicle and its height in the inertial frame are related by $h(t)=h_{0}+z(t)$, where $z(t)$ is the $z$ coordinate of the vehicle in frame $\{I\}$. The dynamic equation for the altitude,

$$
m \ddot{h}=\left(1-2 q_{1}^{2}-2 q_{2}^{2}\right) T-m g,
$$

is derived from the altitude definition and the linear dynamics of the vehicle system represented in (4) and (6). The control law for the thrust $T$ drives the vehicle to a fixed altitude $h^{\star}$ through

$$
T=\frac{m g-k_{1}\left(h-h^{\star}\right)-k_{2} \frac{\dot{h}}{h-h_{0}}}{1-\left(2 q_{1}^{2}+2 q_{2}^{2}\right)}
$$

where $k_{1}$ and $k_{2}$ are positive parameters. The resulting closed-loop altitude dynamics are

$$
m \ddot{h}=-k_{1}\left(h-h^{\star}\right)-\frac{k_{2}}{h-h_{0}} \dot{h},
$$

which amount to a double integrator driven by a PD controller with variable derivative gain.

A subsequent choice of the attitude control law guarantees that the quadrotor never overturns, and thus $2 q_{1}^{2}+2 q_{2}^{2}<1$ for all time, precluding the loss of altitude control through thrust actuation. For now, we take that fact as an assumption and state the following lemma, regarding the altitude control.

Lemma 5: Consider the dynamic system described by the closed-loop system comprising of (15) and (16) with $k_{1}, k_{2}>0$. If the initial conditions verify $z(0)>0$, then the control law is well defined and $z(t)>0$ for all time, even in the presence of attitude observer errors. Additionally, the cascade of the attitude observer and the altitude controller is globally exponentially stable.

Proof: Consider the auxiliar state

$$
\xi=\left(h-h_{0}\right) \exp \left(\frac{1}{k_{2}}\left(m \dot{h}+\int_{0}^{t} k_{1}\left(h(\tau)-h^{\star}\right) \mathrm{d} \tau\right)\right)
$$

and notice that, with the imposed closed-loop dynamics (17), it has a constant value as $\dot{\xi}=0$. Since $\xi(t)=\xi(0)$ is positive and the exponential of a number is always positive, it results that $h(t)-h_{0}>0$ (or equivalently $z(t)>0$ ) for all time and thus collisions with the ground are always avoided.
Global asymptotic stability of $(h, \dot{h})=\left(h^{\star}, 0\right)$ is established from LaSalle's invariance principle and the Lyapunov function

$$
V=\frac{1}{2} \dot{h}^{2}+\frac{1}{2} k_{1}\left(h-h^{\star}\right)^{2}
$$

which has a negative semi-definite time derivative. An additional consequence of the convergence of $(h, \dot{h})$ to $\left(h^{\star}, 0\right)$ and the constancy of the auxiliar state $\xi$ is that $z(t)=$ $h(t)-h_{0}>\epsilon$ and for all time, for some $\epsilon>0$.

Furthermore, LTV system theory asserts that the convergence is indeed exponential. Let $\mathbf{x}=\left[\dot{h} h-h^{\star}\right]^{T}$ and compute

$$
\dot{\mathbf{x}}=\left[\begin{array}{cc}
A(t) & B \\
-C & 0
\end{array}\right] \mathbf{x}=\left[\begin{array}{cc}
-\frac{k_{2}}{z(t)} & -k_{1} \\
1 & 0
\end{array}\right] \mathbf{x}
$$

Let $P=\frac{1}{k_{2}}$ and notice that

$$
A^{T}(t) P+P A(t)=-2 \frac{k_{2}}{k_{1} z(t)}=-Q(t)
$$

with $Q(t)$ bounded as $0<q_{m}<Q(t)<q_{M}$. In these conditions, the LTV system (18) is Uniformly Globally Exponentially Stable [16].

The interconnection of the attitude observer and the altitude subsystem can be regarded as a cascade of two exponentially stable systems [18, Proposition 2.1] with $\frac{k_{2}}{z(t)}$ bounded for all trajectories. In these circumstances, the cascade is also exponentially stable. Finally, impact with the ground is also avoided when the altitude subsystem is perturbed by the orientation errors. This can be established by letting $\Delta_{1}(t)$ be the perturbations due to the estimation errors and considering that the state

$\xi=\left(h-h_{0}\right) \exp \left(\frac{1}{k_{2}}\left(m \dot{h}+\int_{0}^{t} k_{1}\left(h(\tau)-h^{\star}\right)-\Delta_{1}(\tau) \mathrm{d} \tau\right)\right)$

is constant for the perturbed vertical dynamics

$$
m \ddot{h}=-k_{1}\left(h-h^{\star}\right)-\frac{k_{2}}{h-h_{0}} \dot{h}+\Delta_{1}(t) .
$$

\section{B. Stabilization of the Lateral and Longitudinal Dynamics}

The lateral-longitudinal-attitude dynamics of the quadrotor vehicle, with the thrust defined as (16), are described by the following system of equations

$$
\begin{aligned}
& \dot{y}=v_{y}, \quad m \dot{v}_{y}=d(\mathbf{q}) q_{1}+m(\mathbf{q}) q_{2} q_{3}+\delta_{y}, \\
& \dot{x}=v_{x}, \quad m \dot{v}_{x}=-d(\mathbf{q}) q_{2}+m(\mathbf{q}) q_{1} q_{3}+\delta_{x} \text {, } \\
& \dot{q}_{0}=-\frac{1}{2} q^{T} \boldsymbol{\omega}_{B}, \quad \dot{q}=\frac{1}{2}\left(q_{0} I_{4}+S(q)\right) \boldsymbol{\omega}_{B}, \\
& \mathbb{J} \dot{\boldsymbol{\omega}}_{B}=-S\left(\boldsymbol{\omega}_{B}\right) \mathbb{J} \boldsymbol{\omega}_{B}+\mathbf{n},
\end{aligned}
$$

where the components $x, y, v_{x}$ and $v_{y}$ are written in frame $\{I\}$,

$$
\begin{aligned}
d(\mathbf{q}) & =\frac{2 m g q_{0}}{1-\left(2 q_{1}^{2}+2 q_{2}^{2}\right)}, \\
m(\mathbf{q}) & =-\frac{2 m g}{1-\left(2 q_{1}^{2}+2 q_{2}^{2}\right)},
\end{aligned}
$$

and $\delta_{x}, \delta_{y}$ are asymptotically vanishing signals defined as

$$
\begin{aligned}
\delta_{x} & =\frac{2 q_{1} q_{3}+2 q_{0} q_{2}}{1-\left(2 q_{1}^{2}+2 q_{2}^{2}\right)}\left(-k_{1}\left(h-h^{\star}\right)-k_{2} \frac{\dot{h}}{z}\right), \\
\delta_{y} & \left.=\frac{2 q_{2} q_{3}-2 q_{0} q_{1}}{1-\left(2 q_{1}^{2}+2 q_{2}^{2}\right)}\left(-k_{1}\left(h-h^{\star}\right)-k_{2} \frac{\dot{h}}{z}\right)\right) .
\end{aligned}
$$


The control law for the attitude subsystem is chosen as the proportional-differential law

$$
\mathbf{n}=K_{P}\left(\eta-K_{D} \hat{\boldsymbol{\omega}}_{B}\right)
$$

where $K_{P}>0$ and $K_{D}>0$ are design parameters and $\eta=q^{\star}-\hat{q}$ is the attitude error with $q^{\star}$ defined as the virtual control input for the $x-y$ system. The quadrotor attitude subsystem in closed-loop with the control feedback (21) results in the following dynamics, derived for the attitude and angular velocity estimations,

$$
\begin{aligned}
\dot{\hat{q}}_{0} & =-\frac{1}{2} \hat{q}^{T} \hat{\boldsymbol{\omega}}_{B} \\
\dot{\hat{q}} & =\frac{1}{2}\left(\hat{q}_{0} I_{4}+S(\hat{q})\right) \hat{\boldsymbol{\omega}}_{B} \\
\mathbb{J} \dot{\hat{\boldsymbol{\omega}}}_{B} & =-S\left(\hat{\boldsymbol{\omega}}_{B}\right) \mathbb{J} \hat{\boldsymbol{\omega}}_{B}+k_{P}\left(\left(q^{\star}-\hat{q}\right)-k_{D} \hat{\boldsymbol{\omega}}_{B}\right)+\Delta_{2}(t),
\end{aligned}
$$

where the external input

$$
\begin{aligned}
\Delta_{2}(t)=\mathbb{J}\left(\dot{\tilde{\mathbf{b}}}+k_{\omega} \dot{\mathbf{s}}_{\omega}\right)-S\left(\hat{\boldsymbol{\omega}}_{B}\right) \mathbb{J}\left(-\tilde{\mathbf{b}}-k_{\omega} \mathbf{s}_{\omega}\right) \\
-S\left(-\tilde{\mathbf{b}}-k_{\omega} \mathbf{s}_{\omega}\right) \mathbb{J}\left(\hat{\boldsymbol{\omega}}_{B}-\tilde{\mathbf{b}}-k_{\omega} \mathbf{s}_{\omega}\right)
\end{aligned}
$$

includes the errors resulting from the observer measurements and vanishes exponentially fast. According to Proposition 5.7.1 in [13], proper tuning of the torque control law (21) ensures boundedness of the attitude subsystem trajectories and consequent stabilization of the vertical error dynamics, even in the presence of observer errors. In addition, the properly tuned control law ensures that the quadrotor does not overturn for initial attitude and bias estimation errors verifying $\sqrt{\frac{1}{2} \tilde{q}_{0}(0)^{2}+\frac{1}{2} \tilde{\mathbf{b}}(0)^{T} \tilde{\mathbf{b}}(0)}<\epsilon$.

To achieve convergence of the overall system, the virtual control input $q^{\star}$ is generated from the quadrotor position and velocities by a nested saturation control law. Consider the new state variables

$$
\zeta_{1}=\frac{1}{z}\left[\begin{array}{l}
y \\
x
\end{array}\right], \quad \zeta_{2}=\frac{1}{z}\left[\begin{array}{l}
v_{y} \\
v_{x}
\end{array}\right]+\lambda_{1} \sigma\left(\frac{K_{1}}{\lambda_{1}} \zeta_{1}\right)-\frac{v_{z}}{z} \zeta_{1},
$$

where $\sigma(\mathbf{x})=\left(\sigma\left(x_{1}\right), \ldots, \sigma\left(x_{n}\right)\right)$ is a saturation function and $v_{z}=\dot{z}$. Notice that the states $\zeta_{1}$ and $\zeta_{2}$ are readily obtained from $x / z, y / z$ and ${ }^{I} \mathbf{v} / z$, whose estimates can be derived from the camera sensor and attitude estimate.

Fix for $q^{\star}$ the nested saturation structure

$$
q^{\star}=-P_{2} \lambda_{2} \sigma\left(\frac{K_{2}}{\lambda_{2}} \hat{\zeta}_{2}\right)
$$

where

$$
P_{2}=\left[\begin{array}{cc}
1 & 0 \\
0 & -1 \\
0 & 0
\end{array}\right]
$$

and $\hat{\zeta}_{2}$ is the estimate for $\zeta_{2}$ obtained from the image measurements $\mathbf{y}_{i}$ and the attitude estimate $\hat{R}$. The time derivatives of the states are then

$$
\begin{aligned}
\dot{\zeta}_{1}= & \zeta_{2}-\lambda_{1} \sigma\left(\frac{K_{1}}{\lambda_{1}} \zeta_{1}\right) \\
m \dot{\zeta}_{2}= & \frac{D}{z}\left(-P_{2} \lambda_{2} \sigma\left(\frac{K_{2}}{\lambda_{2}} \hat{\zeta}_{2}\right)+\eta\right) \\
& +m K_{1} \sigma^{\prime}\left(\frac{K_{1}}{\lambda_{1}} \zeta_{1}\right) \dot{\zeta}_{1}+\delta_{1}+\delta_{2}+\Delta_{3}
\end{aligned}
$$

where

$$
D=\left[\begin{array}{ccc}
d(\mathbf{q}) & m(\mathbf{q}) q_{3} & 0 \\
m(\mathbf{q}) q_{3} & -d(\mathbf{q}) & 0
\end{array}\right]
$$

the exogenous inputs $\delta_{1}$ and $\delta_{2}$ are given by

$$
\delta_{1}=\left[\begin{array}{l}
\delta_{x} \\
\delta_{y}
\end{array}\right] / z, \quad \delta_{2}=\frac{\left.k_{1}\left(h-h^{\star}\right)+k_{2} \frac{\dot{h}}{z}\right)}{z} \zeta_{1}+m \frac{v_{z}^{2}}{z^{2}} \zeta_{1}-m \frac{v_{z}}{z} \dot{\zeta}_{1}
$$

and the errors due to the attitude estimator are encapsulated in $\Delta_{3}=\frac{D}{z} \tilde{q}$.

From definition (20) and the attitude and vertical controllers, we have the bounds $0<d^{L} \leq d(\mathbf{q}, t) \leq d^{U}$ and $0<z^{L}<z(t)<z^{U}$. The following result is an adaptation of Proposition 5.7.2 and Theorem 5.7.5 in [13] and gives guarantees for the proposed quadrotor stabilization law.

Theorem 6: Let $K_{D}$ be fixed according to Proposition 5.7.1 in [13] and let $K_{i}^{\star}$ and $\lambda_{i}^{\star}, i=1,2$, be such that the following inequalities are satisfied

$$
\frac{\lambda_{2}^{\star}}{K_{2}^{\star}}<\frac{\lambda_{1}^{\star}}{4}, \quad 4 \lambda_{1}^{\star} K_{1}^{\star}<\frac{1}{m} \frac{d^{L}}{z^{U}} \frac{\lambda_{2}^{\star}}{8}, \quad 24 \frac{K_{1}^{\star}}{K_{2}^{\star}}<\frac{1}{6} \frac{d^{L}}{d^{U}} \frac{z^{L}}{z^{U}} .
$$

Then, there exist positive numbers $K_{P}^{\star}$ and $\epsilon^{\star}$ such that, taking

$$
\lambda_{i}=\epsilon^{i} \lambda_{i}^{\star} \text { and } K_{i}=\epsilon K_{i}^{\star}, i=1,2,
$$

for all $K_{P}>K_{P}^{\star}$ and $0<\epsilon \leq \epsilon^{\star}$, the state trajectories of the system (19) in closed-loop with the controller defined by (16), (21) and (22) converge asymptotically to the origin for any initial condition $z(0)>0,\left(x(t), v_{x}(t), y(t), v_{y}(t)\right) \in$ $\mathbb{R}^{4},\left(\hat{q}(0), \hat{\boldsymbol{\omega}}_{B}(0)\right) \in \mathcal{Q} \times \Omega$ and $\left|\tilde{q}_{0}(0)\right|<q_{0}(0)$.

Proof: The proof follows from the arguments in [13] where the statement is proven for constant $z(t)=Z$ and exogenous disturbances $\delta_{2}(t)=0, \Delta(t)=0$. The statement of Theorem 6 is proven by noting that the additional disturbances $\delta_{2}(t)$ and $\Delta(t)$ are asymptotically vanishing. The lateral-longitudinal subsystem does not have finite escape time and the trajectory $\left(\zeta_{1}(t), \zeta_{2}(t)\right)$ exists and is bounded for any $t>0$. Since the disturbance $\delta_{2}(t)$ is asymptotically vanishing, there exists a finite time $T^{\star}$ such that for $t>$ $T^{\star}$ the disturbances are within the bounds for which the convergence of $\left(\zeta_{1}, \zeta_{2}\right)$ to zero is ensured by using gains (24), verifying (23). The remainder of the claims in the theorem statement follows identically from [13].

Gathering the previous results regarding the pan and tilt camera, stabilization of the vertical position, attitude and lateral-longitudinal subsystems, we can now state the following theorem which summarizes the main results of the paper.

Theorem 7: Consider a quadrotor described by the dynamic system (3)-(6) equipped with a pan and tilt camera modeled by (1) with dynamics (14), and apply the set of controllers (14), (16), (21) and (22), using the attitude and rate gyro bias estimator $(10)-(11)$. Then, for any initial condition $z(0)>0,\left(x(t), v_{x}(t), y(t), v_{y}(t)\right) \in \mathbb{R}^{4}$, $\left(\hat{q}(0), \hat{\boldsymbol{\omega}}_{B}(0)\right) \in \mathcal{Q} \times \Omega$ and $\left|\tilde{q}_{0}(0)\right|<q_{0}(0)$ such that the landmarks are visible in the image plane of the camera, the vehicle's position, attitude, velocities converge asymptotically to ${ }^{I} \mathbf{p}_{B}=\left[\begin{array}{lll}0 & 0 & h^{\star}-h_{0}\end{array}\right]^{T},{ }_{B}^{I} R=I_{3}, \mathbf{v}_{B}=0, \boldsymbol{\omega}_{B}=$ 0 , respectively, whereas the camera's velocity and image coordinates converge to $\boldsymbol{\omega}_{C}=0$ and $\overline{\mathbf{y}}=0$, respectively.

Proof: The stated result follows immediately from Theorems 6 and 3. Theorem 6 states that convergence of the vehicle position and velocity to zero is achieved, even in the presence of attitude estimation errors. Convergence of the 
landmarks' centroid image coordinates to zero is achieved if the vehicle velocity and bias error converge to zero, which is guaranteed by Theorem 3 .

\section{SIMULATION RESULTS}

In this section, we present the results from a simulation run of the proposed control architecture. At the initial configuration the quadrotor is assumed at rest. The camera points towards a set of landmarks that are visible and the centroid of the landmarks is not coincident with origin of the image plane. The objective of the simulation is to hover the quadrotor over the centroid of the landmarks at a reference vertical position. The vehicle parameters are $m=1 \mathrm{~kg}$, $\mathbb{J}=0.5 I_{3} \mathrm{~kg} \mathrm{~m}^{2}, \lambda_{1}=10, \lambda_{2}=0.3, K_{1}=0.3, K_{2}=0.3$, $K_{P}=10, K_{D}=.5, k_{1}=0.1$ and $k_{2}=6$.

Figure 3 presents the time evolution of the quadrotor position error expressed in inertial coordinates. We can verify that the error converges from the initial $\mathbf{e}=\left[\begin{array}{lll}8-6 & -3\end{array}\right]^{T} \mathrm{~m}$ to zero and is negligible after about 20 seconds.

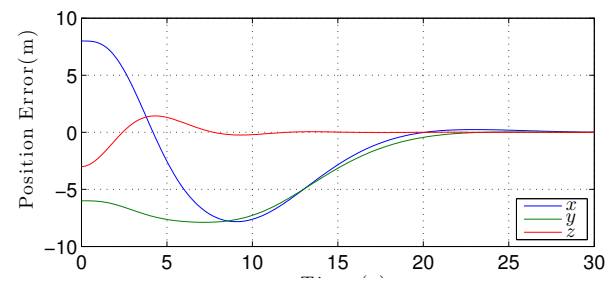

Fig. 3. Inertial position error of the quadrotor.

The position of the landmarks' centroid in the image plane is displayed in Figure 4. The centroid $\overline{\mathbf{y}}$ converges asymptotically to the origin as the velocity of the quadrotor converges asymptotically to zero. The disturbance effect of the quadrotor linear velocity and observer errors on the time evolution of $\overline{\mathbf{y}}$ can be observed in the figure by noting that the convergence to the origin is not monotonic.

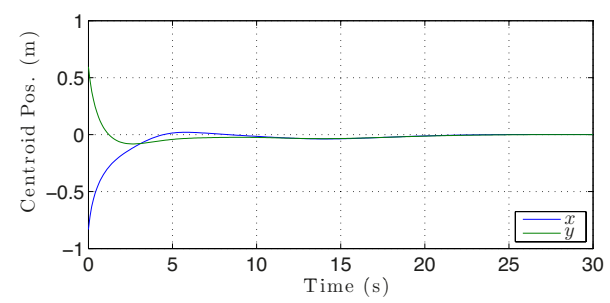

Fig. 4. Landmarks' centroid position in image coordinates.

\section{CONCLUSIONS}

This paper proposed a cascaded architecture comprising a nonlinear attitude observer and a nonlinear controller for the stabilization of a quadrotor vehicle based on image measurements of a set of landmarks obtained from a pan and tilt camera and biased rate gyros. The vehicle was stabilized vertically to a given altitude with a PD control law based on image measurements and a vertical position sensor. The lateral-longitudinal stabilization was achieved with a nested saturation control law using feedback of the image measurements, estimated body attitude and angular rate. Both controllers were shown to be ISS with respect to the attitude and rate gyros bias estimation error, which allows for the closed-loop stability of the cascaded architecture. During the whole stabilization procedure the pan and tilt camera was actuated so as to keep the image of the landmarks' centroid at the center of the image plane. Simulation results exhibited good performance and attested the applicability of the proposed technique.

\section{REFERENCES}

[1] F. Chaumette and S. Hutchinson, "Visual servo control, part I: Basic approaches," IEEE Robotics and Automation Magazine, vol. 13, no. 4, pp. 82-90, December 2006.

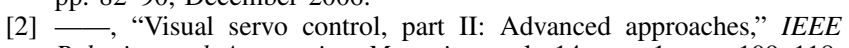
Robotics and Automation Magazine, vol. 14, no. 1, pp. 109-118, March 2007.

[3] N. Cowan, J. Weingarten, and D. Koditschek, "Visual servoing via navigation functions," Robotics and Automation, IEEE Transactions on, vol. 18, no. 4, pp. 521-533, Aug 2002.

[4] R. Cunha, C. Silvestre, J. ao Hespanha, and A. P. Aguiar, "Visionbased control for rigid body stabilization," Automatica, vol. 47, no. 5, pp. $1020-1027,2011$.

[5] E. Malis and F. Chaumette, "Theoretical improvements in the stability analysis of a new class of model-free visual servoing methods," Robotics and Automation, IEEE Transactions on, vol. 18, no. 2, pp. 176-186, Apr 2002.

[6] A. Aguiar and J. Hespanha, "Minimum-energy state estimation for systems with perspective outputs," Automatic Control, IEEE Transactions on, vol. 51, no. 2, pp. 226-241, Feb. 2006.

[7] Yi Ma, S. Soatto, J. Kosecka, and S. Sastry, An Invitation to 3-D Vision From Images to Geometric Models. Springer, 2004, volume 26 of Interdisciplinary Applied Mathematics.

[8] H. Rehbinder and B. K. Ghosh, "Pose estimation using line-based dynamic vision and inertial sensors," IEEE Transactions on Automatic Control, vol. 48, no. 2, pp. 186-199, 2003.

[9] J. Lobo and J. Dias, "Vision and inertial sensor cooperation using gravity as a vertical reference," IEEE Transactions on Pattern Analysis and Machine Intelligence, vol. 25, no. 12, pp. 1597 - 1608, Dec. 2003.

[10] A. I. Mourikis, N. Trawny, S. I. Roumeliotis, A. E. Johnson, A. Ansar, and L. Matthies, "Vision-aided inertial navigation for spacecraft entry, descent, and landing," IEEE Transaction on Robotics, vol. 25, no. 2, pp. 264-280, 2009.

[11] E. Frazzoli, M. Dahleh, and E. Feron, "Trajectory tracking control design for autonomous helicopters using a backstepping algorithm," in American Control Conference, 2000. Proceedings of the 2000, vol. 6, 2000, pp. 4102-4107 vol.6.

[12] D. Cabecinhas, R. Cunha, and C. Silvestre, "Trajectory tracking control for quadrotors," European Control Conference, 2009.

[13] A. Isidori, L. Marconi, and A. Serrani, Robust Autonomous Guidance. Springer, 2003.

[14] L. Marconi and R. Naldi, "Robust full degree-of-freedom tracking control of a helicopter," Automatica, vol. 43, no. 11, pp. 1909 - 1920, 2007.

[15] S. Brás, R. Cunha, J. Vasconcelos, C. Silvestre, and P. Oliveira, "A nonlinear attitude observer based on active vision and inertial measurements," Robotics, IEEE Transactions on, vol. 27, no. 4, pp. 664-677, Aug. 2011.

[16] A. Loría and E. Panteley, "Uniform exponential stability of linear timevarying systems: revisited," Systems and Control Letters, vol. 47, no. 1, pp. 13-24, Sep. 2002.

[17] S. P. Bhat and D. S. Bernstein, "A topological obstruction to continuous global stabilization of rotational motion and the unwinding phenomenon," Systems and Control Letters, vol. 39, no. 1, pp. 63-70, Jan. 2000.

[18] S. Sastry and A. Isidori, "Adaptive control of linearizable systems," Automatic Control, IEEE Transactions on, vol. 34, no. 11, pp. 1123 -1131 , nov 1989. 